\title{
Radar Cross-Section Study of Cylindrical Cavity- Backed Apertures with Outer or Inner Material Coating: The Case of...
}

Article in IEEE Transactions on Antennas and Propagation · December 1993

DOI: $10.1109 / 8.267355 \cdot$ Source: IEEE Xplore

CITATIONS

35

3 authors:

\section{Dilek Colak}

King Faisal Specialist Hospital and Research Ce...

88 PUBLICATIONS 1,016 CITATIONS

SEE PROFILE

\section{Ayhan Altintas}

Bilkent University

132 PUBLICATIONS 721 CITATIONS

SEE PROFILE
READS

52

\section{Alexander Nosich}

Institute of Radio-Physics and Electronics of th...

375 PUBLICATIONS $\quad 2,920$ CITATIONS

SEE PROFILE

Some of the authors of this publication are also working on these related projects: 


\title{
Radar Cross-Section Study of Cylindrical Cavity-Backed Apertures with Outer or Inner Material Coating: The Case of $E$-Polarization
}

\author{
Dilek Çolak, Alexander I. Nosich, and Ayhan Altıntaş, Member, IEEE
}

\begin{abstract}
A dual-series-based solution is obtained for the scattering of an E-polarized plane wave from a cavity-backed aperture which is formed by a slitted infinite circular cylinder coated with absorbing material. The material coating can be done on the inner or outer surface of the cylinder. For both cases, numerical results are presented for the radar cross section and comparisons are given for two different realistic absorbing materials. The radar cross-section results are also given for the aspect angle of the screen. Finally, the dependence of radar cross section on the thickness of the absorbing layer is presented.
\end{abstract}

\section{INTRODUCTION}

$\mathrm{C}$ AVITY-BACKED apertures (CBA) are encountered as parts of any airborne or spaceborne radar targets. The most familiar CBAs are, probably, air inlets and engine tubes, known to contribute a great deal to the radar cross section of jet aircraft. What is even more dangerous, the CBAs are famous for the internal resonances, which can easily result in recognizing the shape of a target. More often than not, these effects are considered to be undesirable and are to be suppressed. To this end, the walls of the cavity are covered with some lossy material.

To simulate the scattering from these CBA geometries, two- and three-dimensional (2D and 3D) models of openended waveguide-type cavities are usually employed. Absorption for thin coatings is generally modeled by introducing boundary conditions of impedance type. In the papers [1]-[6], the scattering from such cavities is treated by various approximate asymptotic approaches, such as GTD, UTD, bouncing ray, and hybrid methods (modal and ray approaches). The simplicity and physical appeal of these high-frequency approaches are not complemented by the clear limits of accuracy. Besides, it is principally difficult to take into account various parts of the scatterer and their interaction with uniform accuracy. Also, the high-frequency approaches mentioned fail for cavities with

Manuscript received January 15, 1993; revised June 18, 1993. Work partially supported by NATO-SFS, TU-MIMIC, and the Scientific and Technical Research Council of Turkey (TÜBITAK).

The authors are with the Department of Electrical and Electronics Engineering, Bilkent University, 06533 Bilkent, Ankara, Turkey.

IEEE Log Number 9213073. dimensions comparable to the wavelength. A WienerHopf-based approach to solve similar 2D problems for unloaded and loaded rectangular CBA [7] is free of these difficulties, but it becomes cumbersome if the walls of the cavity are covered with absorbers. In a recent study [8], the multiple parameter perturbation analysis has been applied to the slitted loaded cavity problem comprising two eccentric circular cylinders. The discussion on the comparative advantages of different techniques is still continuing (see [9] and the list of references).

For certain canonical geometries, there exists an accurate approach of analytical-numerical nature which ensures any desired accuracy of the obtained results. This is the dual-series-based Riemann-Hilbert (RHP) approach, which has been under intensive study in the former USSR since the 1960s [10]. In the $1970 \mathrm{~s}$ and 1980s a large amount of results have been obtained on free-space scattering from open screens and collections of screens (see [11] and the cited literature). In the West the dual-seriesbased approach was exploited by Ziolkowski [12]-[14]. It was unfortunate that a numerical error was present in [13] for the $E$ case (reported in [15]), which obviously should not discredit the method. Actually, correct RHP-based radar cross-section (RCS) analysis results were published in [16], but remained unknown for western readers. In the present study, the dual-series-based RHP technique has been extended to solve the problems of CBAs with nonhomogeneous space inside or outside. Our canonical geometry is a circular shell formed by a zero-thickness, perfectly conducting screen having an opening. Arbitrary thin lossy material can be introduced as a concentric layer on either inner or outer surface of the shell. We do realize that this $2 \mathrm{D}$ model geometry is far from a real jet inlet. Nevertheless, such a scatterer exhibits resonant behavior of quite a general nature. Studying this behavior and the effect of absorber in detail, one can judge the more realistic geometries.

The main advantage of the dual-series-based approach is that it is based on the idea of partial analytical inversion of the scattering operator. Final matrix equations are proven to be of the Fredholm second kind, so the solution exists and can be approximated through truncation. What is also important is that the method is equally effective for 
any angular width of the shell from 0 to $2 \pi$. The size of the matrix is determined by the electrical radius of curvature, and fairly large structures can be treated accurately. The numerical data obtained can obviously bring a better understanding of the scattering behavior of loaded cavities. It can also serve as reference data for checking numerical codes for more complicated scatterers, e.g., solved by method of moments [17], [18].

The remainder of the paper is organized as follows. In Section II, we present the formulation of the problem. The dual series equations are derived and then solved via the RHP approach in Sections III and IV, respectively. Sample numerical results are presented in Section V. Finally, some conclusions are given in Section VI.

Throughout this paper the time dependence $e^{-i w t}$ has been assumed and suppressed.

\section{FORMULATION}

The geometries analyzed in this paper are shown in Figs. 1 and 2. The electric field vector of the incident plane wave is taken to be parallel to the axis of the cylinder, and the axial component of the magnetic field is taken as zero (i.e., TM with respect to the axis of the cylinder). This $E$-polarized plane wave is assumed to be normally incident on the cylinder; hence, the problem is two dimensional. The coordinate system $(r, \varphi, z)$ is coaxial with the cylinder. The screen which has a radius of $a$ is taken to be in the interval of $\theta<\left|\varphi-\varphi_{0}\right| \leq \pi$. The angular width of the slit is $2 \theta$ and the angle between the center of the slit and the $x$ axis is $\varphi_{0}$. The cylindrical cavity is coated with an absorbing material with a thickness $t$. The radius $b$ is at either $a-t$ or $a+t$ depending on whether the coating is on the inside or on the outside, respectively. The relative permittivity and permeability of the absorbing material are $\epsilon_{r}$ and $\mu_{r}$, respectively. Our objective is to analyze the radar scattering behavior of this geometry for various frequencies. The problem is scalar, so the total field can be characterized by the single $E_{z}$ component.

First, consider the geometry shown in Fig. 1. The total field can be expressed as

$$
E_{z}(\boldsymbol{r})= \begin{cases}E_{z}^{\mathrm{in}}(\boldsymbol{r})+E_{z}^{\mathrm{sc}}(\boldsymbol{r}), & r>a, \\ E_{z}^{\mathrm{sc}}(\boldsymbol{r}), & b<r<a, \\ E_{z}^{\mathrm{sc}}(\boldsymbol{r}), & r<b,\end{cases}
$$

where $E_{z}^{\text {in }}$ and $E_{z}^{\text {sc }}$ stand for incident and scattered fields, respectively.

The scattered field $E_{z}^{\text {sc }}$ satisfies the 2D Helmholtz equations:

$$
\begin{array}{rlrl}
\left(\nabla^{2}+k_{0}^{2}\right) E_{z}^{\mathrm{sc}}(\boldsymbol{r}) & =0, & & r>a, r<b, \\
\left(\nabla^{2}+k_{0}^{2} \epsilon_{r} \mu_{r}\right) E_{z}^{\mathrm{sc}}(\boldsymbol{r}) & =0, & b<r<a,
\end{array}
$$

where $k_{0}=\omega \sqrt{\mu_{0} \epsilon_{0}}$ is the free-space wave number and $\nabla^{2}$ is the Laplacian operator.

In addition, we impose the following boundary conditions: First, the continuity of $E_{z}$ on the closed contours

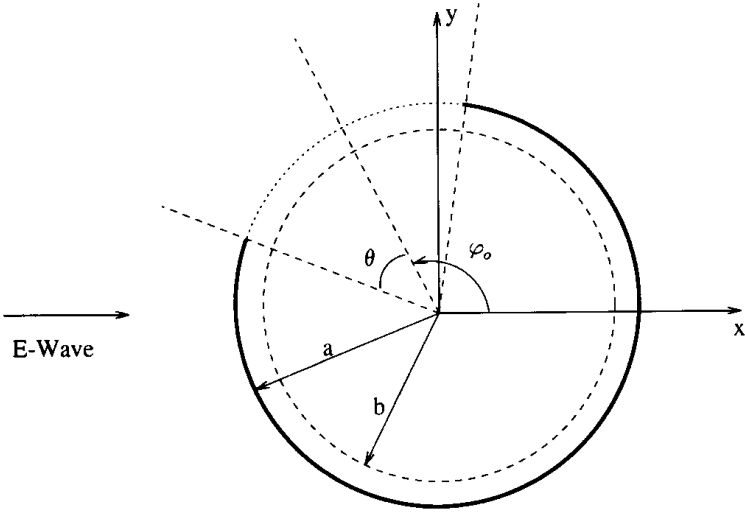

Fig. 1. $E$-wave scattering by an inner-coated cavity-backed aperture.

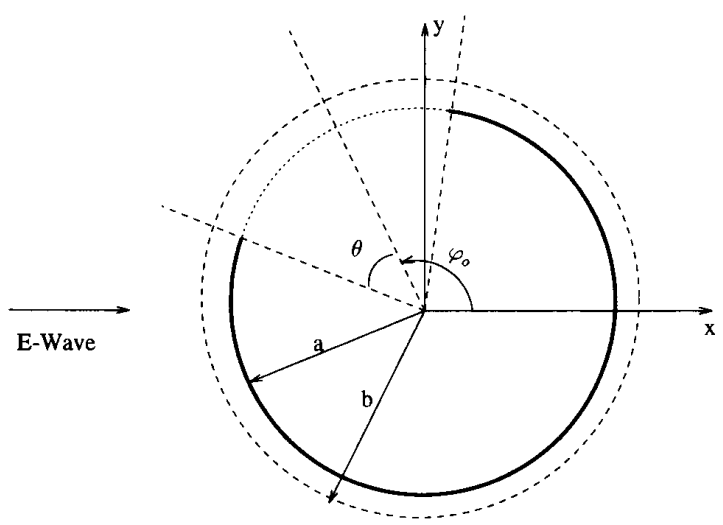

Fig. 2. $E$-wave scattering by an outer-coated cavity-backed aperture.

$r=a$ as well as $r=b$, and the continuity of $H_{\varphi}$ on $r=b$. Second, the continuity of $H_{\varphi}$ in the aperture region and vanishing $E_{z}$ on the screen, at $r=a$.

Because of the sharp edges of the screen, the field should satisfy certain edge conditions. These conditions are satisfied by the requirement that the total electrical and magnetic energy stored inside any finite neighborhood of the edge must be finite. For the polarization considered, the magnetic field is proportional to $\nabla E_{z}$, so this requirement is given by

$$
\int_{B}\left(k_{0}^{2}\left|E_{z}^{\mathrm{sc}}\right|^{2}+\left|\nabla E_{z}^{\mathrm{sc}}\right|^{2}\right) d r<\infty
$$

where $B$ is any bounded domain around the edge. Finally, the Sommerfeld radiation condition

$$
E^{\mathrm{sc}}(\boldsymbol{r}) \sim \Phi(\varphi)\left(\frac{2}{i \pi k_{0} r}\right)^{1 / 2} e^{i k_{0} r}
$$

should be satisfied far from the scatterer as $r \rightarrow \infty$. 


\section{Derivation of Dual Series Equations}

The scattered field expansions in three regions of Fig. 1 are assumed to be

$$
E_{z}^{\mathrm{sc}}=\sum_{n=-\infty}^{\infty}\left\{\begin{array}{ll}
A_{n} H_{n}\left(k_{0} r\right), & r>a \\
B_{n} J_{n}(k r)+C_{n} H_{n}(k r), & b<r<a \\
D_{n} J_{n}\left(k_{0} r\right), & r<b
\end{array}\right\} e^{i n \varphi},
$$

where $k=k_{0} \sqrt{\mu_{r} \epsilon_{r}}, J_{n}$ and $H_{n}$ represent the Bessel and Hankel functions of first kind and order $n$, respectively.

The incident plane wave of unit amplitude has the Fourier expansion

$$
E_{z}^{\text {in }}=e^{i k_{0} x}=e^{i k_{0} r \cos \varphi}=\sum_{n=-\infty}^{\infty} i^{n} J_{n}\left(k_{0} r\right) e^{i n \varphi} .
$$

The number of unknown coefficients in (5) can be reduced by applying boundary conditions that are valid on the closed contour; that is, the continuity of $E_{z}$ at $r=a$ and $r=b$ as well as the continuity of $H_{\varphi}$ at $r=b$. Hence

$$
\begin{gathered}
B_{n}=\frac{i^{n} J_{n}\left(k_{0} a\right)+H_{n}\left(k_{0} a\right) A_{n}}{J_{n}(k a) \xi_{n}-H_{n}(k a) \eta_{n}} \xi_{n}, \\
C_{n}=-\frac{i^{n} J_{n}\left(k_{0} a\right)+H_{n}\left(k_{0} a\right) A_{n}}{J_{n}(k a) \xi_{n}-H_{n}(k a) \eta_{n}} \eta_{n},
\end{gathered}
$$

and

$$
D_{n}=\frac{2 i}{\pi k_{0} b \mu_{r}} \frac{i^{n} J_{n}\left(k_{0} a\right)+H_{n}\left(k_{0} a\right) A_{n}}{J_{n}(k a) \xi_{n}-H_{n}(k a) \eta_{n}}
$$

are obtained. In (7), (8), and (9),

$$
\begin{aligned}
& \xi_{n}=\sqrt{\frac{\epsilon_{r}}{\mu_{r}}} H_{n}^{\prime}(k b) J_{n}\left(k_{0} b\right)-H_{n}(k b) J_{n}^{\prime}\left(k_{0} b\right), \\
& \eta_{n}=\sqrt{\frac{\epsilon_{r}}{\mu_{r}}} J_{n}^{\prime}(k b) J_{n}\left(k_{0} b\right)-J_{n}(k b) J_{n}^{\prime}\left(k_{0} b\right),
\end{aligned}
$$

where the prime denotes derivative with respect to the argument.

The zero value of $E_{z}$ on the screen and the continuity of $H_{\varphi}$ in the aperture lead to dual series equations for the expansion coefficients

$$
\begin{aligned}
\sum_{n=-\infty}^{\infty} x_{n} \gamma_{n} e^{i n \varphi} & =-\sum_{n=-\infty}^{\infty} d_{n} e^{i n \varphi}, \quad\left|\varphi-\varphi_{0}\right|<\theta, \\
\sum_{n=-\infty}^{\infty} x_{n} e^{i n \varphi} & =0, \quad \theta<\left|\varphi-\varphi_{0}\right| \leq \pi,
\end{aligned}
$$

where

$$
\begin{aligned}
& x_{n}=i^{n} J_{n}\left(k_{0} a\right)+H_{n}\left(k_{0} a\right) A_{n}, \\
& d_{n}=-\frac{2 i^{n+1}}{\pi k_{0} a H_{n}\left(k_{0} a\right)},
\end{aligned}
$$

and

$$
\gamma_{n}=\frac{H_{n}^{\prime}\left(k_{0} a\right)}{H_{n}\left(k_{0} a\right)}-\sqrt{\frac{\epsilon_{r}}{\mu_{r}}} \frac{J_{n}^{\prime}(k a) \xi_{n}-H_{n}^{\prime}(k a) \eta_{n}}{J_{n}(k a) \xi_{n}-H_{n}(k a) \eta_{n}} .
$$

\section{DuAl-Series-Based Solution}

Investigating the asymptotic behavior of $\gamma_{n}$ as $|n| \rightarrow \infty$, based on the corresponding expressions for cylindrical functions [19], we find

$$
\gamma_{n} \sim-\frac{|n|}{k_{0} a}\left(1+\frac{\beta_{n}}{\mu_{r}}\right)
$$

where

$$
\beta_{n}=1+2 \frac{\mu_{r}-1}{\mu_{r}+1}\left(\frac{b}{a}\right)^{2 n}\left[1-\frac{\mu_{r}-1}{\mu_{r}+1}\left(\frac{b}{a}\right)^{2 n}\right]^{-1} .
$$

For $b \neq a$, the dominant term in (16) is the first term, which is 1 . The remaining terms decrease very fast as $n$ increases.

Adding and subtracting the asymptotic expression (15) from $\gamma_{n}$ in (10), we get the result

$$
\begin{array}{r}
\sum_{n=-\infty}^{\infty} x_{n}|n| e^{i n \varphi}=\sum_{n=-\infty}^{\infty}\left(x_{n} \Delta_{n}+\frac{\mu_{r} k_{0} a}{\mu_{r}+1} d_{n}\right) e^{i n \varphi}, \\
\left|\varphi-\varphi_{0}\right|<\theta,
\end{array}
$$

where

$$
\Delta_{n}=\frac{\mu_{r} k_{0} a}{\mu_{r}+1} \gamma_{n}+|n|
$$

and $x_{n}$ and $d_{n}$ are given in (12) and (13), respectively.

Equations (17) and (11) form canonical dual-series equations. This dual-series system can be solved by converting into the Riemann-Hilbert problem [10]-[12]. Assuming that the series (11) is term-by-term differentiable, we replace it with the derivative with respect to $\varphi$. The termwise differentiation can be justified as described in [11]. Denoting $\psi=\varphi-\varphi_{0}$, we have

$$
\begin{aligned}
& \sum_{n=-\infty}^{\infty} \tilde{x}_{n}|n| e^{i n \psi}=F\left(e^{i \psi}\right), \quad|\psi|<\theta, \\
& \sum_{n=-\infty}^{\infty} \tilde{x}_{n} n e^{i n \psi}=0, \quad \theta<|\psi| \leq \pi, \\
& \sum_{n=-\infty}^{\infty}(-1)^{n} \tilde{x}_{n}=0,
\end{aligned}
$$

where $\tilde{x}_{n}=x_{n} e^{i n \varphi_{0}}$. The last equation is obtained by substituting $\varphi=\pi+\varphi_{0}$ into (11) to account for the elimination of the constant term due to differentiation. The function on the right-hand side of (19a) has a Fourier expansion as

$$
F\left(e^{i \psi}\right)=\sum_{n=-\infty}^{\infty} f_{n} e^{i n \psi}
$$


where the coefficients are given as

$$
f_{n}=\left(x_{n} \Delta_{n}+\frac{\mu_{r} k_{0} a}{\mu_{r}+1} d_{n}\right) e^{i n \varphi_{0}} .
$$

By introducing functions $X^{ \pm}$of complex variable $z=$ $|z| e^{i \psi}$ such that

$$
X(z)= \begin{cases}X^{+}(z)=\sum_{n>0} \tilde{x}_{n} n z^{n}, & |z| \leq 1, \\ X^{-}(z)=-\sum_{n<0} \tilde{x}_{n} n z^{n}, & |z| \geq 1,\end{cases}
$$

and using (19a) and (19b), the functional equation valid on the whole unit circle $|z|=1$,

$$
X^{+}\left(e^{i \psi}\right)+A X^{-}\left(e^{i \psi}\right)=B,
$$

is obtained with

$$
\begin{aligned}
& A(\psi)= \begin{cases}+1, & |\psi|<\theta, \\
-1, & \theta<|\psi| \leq \pi,\end{cases} \\
& B(\psi)= \begin{cases}F\left(e^{i \psi}\right), & |\psi|<\theta, \\
0, & \theta<|\psi| \leq \pi .\end{cases}
\end{aligned}
$$

This is the equation which is known to constitute RHP. To arrive at the exact solution of (23), it is necessary to restrict the behavior of the unknown function $X(z)$ at infinity and at the end points of the screen. One may see from (19) and (21) that the frequency dependence is contained only in the $F\left(e^{i \psi}\right)$ term. Assuming that $F\left(e^{i \psi}\right)$ is known, (23) forms a static problem $(k=0)$ for $X(z)$. However, at static limit, the incident plane wave constitutes a supersposition of two cross-polarized constant fields: electric $E_{z}$ and magnetic $H_{y}$. The perfectly conducting cylinder does not perturb the axial electric field, but it does perturb the transverse magnetic field. So, function $X(z)$ corresponds to the perturbation of $H_{y}$ by the presence of the screen which then vanishes as $|z| \rightarrow \infty$. From (3), the field behavior at the edges for $E_{z}$ and $H_{\varphi}$ will be like $(|\psi|-\theta)^{1 / 2}$ and $(|\psi|-\theta)^{-1 / 2}$, respectively. So, $X(z)$ has a square-root singularity at the edges of the screen.

Further we follow [11] and use the Riemann-Hilbert problem solution in the form

$$
\tilde{x}_{m}=\sum_{n=-\infty}^{\infty} f_{n} T_{m n}(\cos \theta),
$$

where $f_{n}$ is the Fourier expansion coefficient given in (21). $T_{m n}(u)$ is related to the Legendre polynomials $\left(P_{m}\right.$ and $P_{n}$ ) through the expressions

$$
T_{m n}(u)= \begin{cases}Q_{m n}(u), & m \neq 0, \\ Q_{n 0}(u), & m=0, n \neq 0, \\ -\ln [(1+u) / 2], & m=n=0\end{cases}
$$

where

$$
\begin{aligned}
Q_{m n}(u)= & \frac{1}{2(m-n)}\left[P_{m-1}(u) P_{n}(u)-P_{m}(u) P_{n-1}(u)\right], \\
& m \neq n \\
Q_{m m}(u)= & \frac{\operatorname{sgn}(m)}{2 m} \sum_{s=0}^{|m|} q_{|m|-s}(u) P_{|m|-s-1}(u), \\
q_{0}(u)= & 1, \quad q_{1}(u)=-u, \ldots, \\
& q_{s}(u)=P_{s}(u)-2 u P_{s-1}(u)+P_{s-2}(u),
\end{aligned}
$$

and we make use of the relation $P_{-s-1}(u)=P_{s}(u)$.

This form of the solution of the dual-series equations is simpler than the solution in [10], [12]-[14], since it does not require the separation of equations for $m=0$ and $m \neq 0$ parts.

By defining

$$
\rho_{n}=A_{n} / J_{n}\left(k_{0} a\right),
$$

one can write (24) as

$$
\rho_{m}=\sum_{n=-\infty}^{\infty} K_{m n} \rho_{n}+S_{m}, \quad m=0, \pm 1, \ldots,
$$

where

$$
\begin{aligned}
K_{m n}= & \frac{H_{n}\left(k_{0} a\right) J_{n}\left(k_{0} a\right)}{H_{m}\left(k_{0} a\right) J_{m}\left(k_{0} a\right)} \Delta_{n} W_{m n}, \\
S_{m}= & \frac{-i^{m}}{H_{m}\left(k_{0} a\right)}+\frac{1}{H_{m}\left(k_{0} a\right) J_{m}\left(k_{0} a\right)} \\
& \cdot \sum_{n=-\infty}^{\infty} i^{n}\left[J_{n}\left(k_{0} a\right) \Delta_{n}-\frac{2 i \mu_{r}\left(\mu_{r}+1\right)^{-1}}{\pi H_{n}\left(k_{0} a\right)}\right] W_{m n},
\end{aligned}
$$

and

$$
W_{m n}=e^{i(n-m) \varphi_{0}} T_{m n}(\cos \theta) .
$$

The coefficients $W_{m n}$ contain all the information about the angular geometry of the screen as functions of $\theta$ and $\varphi_{0}$.

Expression (27) can be written as a single operator equation

$$
(I-K) \rho=S \text {, }
$$

where $\rho=\left\{\rho_{n}\right\}_{n=-\infty}^{\infty}, I$ is identity matrix, and $K=$ $\left\{K_{m n}\right\}_{m, n=-\infty}^{\infty}$. Operator $K$ can be shown (see [11]) to be compact in the Hilbert space of $l_{2}\left(\rho \in l_{2}\right.$ if $\sum_{n=-\infty}^{\infty}\left|\rho_{n}\right|^{2}$ $<\infty)$. Besides, vector $S=\left\{S_{m}\right\}_{m=-\infty}^{\infty} \in l_{2}$ as well. This means that (31) is a regularized operator equation, and therefore well known Fredholm's theorems are valid [22]: solution $\rho$ does exist and is unique. Moreover, any solution of (31) can be shown (see [11]) to satisfy

$$
\sum_{n=-\infty}^{\infty}\left|\rho_{n}\right|^{2}|n+1|<\infty
$$

as $\rho_{n}$ decays as $O\left(n^{-3 / 2}\right)$ when $|n| \rightarrow \infty$. Hence, (32) ensures the validity of the edge condition (3) [11]. Further, this solution can be approximated with any desired accu- 
racy by means of truncation of the matrix $K=$ $\left\{K_{m n}{ }_{m, n=-\infty}^{\infty}\right.$ and vector $S=\left\{S_{m}\right\}_{m=-\infty}^{\infty}$ for all $|m|,|n|>$ $N_{\mathrm{tr}}$. The sequence of approximate solutions is guaranteed to converge to the exact solution for any $k_{0} a, b / a, \epsilon_{r}, \mu_{r}, \theta, \varphi_{0}$ as $N_{\mathrm{tr}} \rightarrow \infty$, which is not the general case in the method of moments. In practice, the simple numerical rule has been verified. To provide an accuracy of $0.1 \%$, we had to take $N_{\mathrm{tr}}=$ the integer part of $\left(k_{0} a\right)+15$. It is noted that all the field coefficients in (5), namely, $A_{n}, B_{n}, C_{n}$, and $D_{n}$ are calculated using equations (26), (7), (8), and (9), respectively.

We treat the second problem (for outer covering) in a similar way. In (5), we replace $a$ s with $b \mathrm{~s}$; afterward, the same procedure is followed. The resultant infinite system of linear equations is formally the same as in the previous problem (27). It is rewritten for convenience as

$$
\rho_{m}=\sum_{(n)} K_{m n} \rho_{n}+S_{m}, \quad m=0, \pm 1, \ldots,
$$

where now

$$
K_{m n}=\frac{J_{n}\left(k_{0} a\right)}{J_{m}\left(k_{0} a\right)} \frac{H_{n}(k a) \xi_{n}-J_{n}(k a) \eta_{n}}{H_{m}(k a) \xi_{m}-J_{m}(k a) \eta_{m}} \Delta_{n} W_{m n}
$$

and the coefficients are

$$
\begin{aligned}
& \xi_{n}=J_{n}(k b) H_{n}^{\prime}\left(k_{0} b\right)-\sqrt{\frac{\epsilon_{r}}{\mu_{r}}} J_{n}^{\prime}(k b) H_{n}\left(k_{0} b\right), \\
& \eta_{n}=H_{n}(k b) H_{n}^{\prime}\left(k_{0} b\right)-\sqrt{\frac{\epsilon_{r}}{\mu_{r}}} H_{n}^{\prime}(k b) H_{n}\left(k_{0} b\right),
\end{aligned}
$$

and

$$
\Delta_{n}=-\frac{\mu_{r} k_{0} a}{\mu_{r}+1} \gamma_{n}+|n|
$$

where

$$
\gamma_{n}=\frac{J_{n}^{\prime}\left(k_{0} a\right)}{J_{n}\left(k_{0} a\right)}-\sqrt{\frac{\epsilon_{r}}{\mu_{r}}} \frac{H_{n}^{\prime}(k a) \xi_{n}-J_{n}^{\prime}(k a) \eta_{n}}{H_{n}(k a) \xi_{n}-J_{n}(k a) \eta_{n}} .
$$

Coefficient $S_{m}$ in (33) is now given by

$$
\begin{aligned}
S_{m}= & J_{m}^{-1}\left(k_{0} a\right)\left[H_{m}(k a) \xi_{m}-J_{m}(k a) \eta_{m}\right]^{-1} \\
& \cdot\left\{-i^{m}\left[H_{m}(k a) s_{2 m}-J_{m}(k a) s_{4 m}\right]\right. \\
& +\sum_{n=-\infty}^{\infty} i^{n}\left\{\left[H_{n}(k a) s_{2 n}-J_{n}(k a) s_{4 n}\right] \Delta_{n}\right. \\
& \left.\left.+8 i \alpha^{-1}\left[H_{n}(k a) \xi_{n}-J_{n}(k a) \eta_{n}\right]^{-1}\right\} W_{m n}\right\},
\end{aligned}
$$

where

$$
\begin{aligned}
\alpha & =\pi^{3}\left(k_{0} b\right)^{2} \mu_{r}\left(\mu_{r}+1\right), \\
s_{2 n} & =J_{n}(k b) J_{n}^{\prime}\left(k_{0} b\right)-\sqrt{\frac{\epsilon_{r}}{\mu_{r}}} J_{n}^{\prime}(k b) J_{n}\left(k_{0} b\right), \\
s_{4 n} & =H_{n}(k b) J_{n}^{\prime}\left(k_{0} b\right)-\sqrt{\frac{\epsilon_{r}}{\mu_{r}}} H_{n}^{\prime}(k b) J_{n}\left(k_{0} b\right) .
\end{aligned}
$$

The second problem has the same type of operator equation as the previous one.

\section{NUMERICAL RESUlTS AND DisCUSSION}

Numerical results are obtained for RCS behavior of a CBA which is coated either from inside or from outside with absorbing materials. The associated formula for RCS is given as

$$
\sigma_{b s}=\lim _{r \rightarrow \infty} 2 \pi r \frac{\left|E^{\mathrm{sc}}(r, \pi)\right|^{2}}{\left|E^{\mathrm{in}}\right|^{2}},
$$

which can be written in terms of expansion coefficients as

$$
\sigma_{b s}=\frac{4}{k_{0}}\left|\sum_{-N_{\mathrm{tr}}}^{N_{\mathrm{tr}}} \rho_{n} i^{n} J_{n}\left(k_{0} a\right)\right|^{2} .
$$

We normalized RCS with respect to $\pi a$ which is the geometrical optics value for the perfectly conducting closed circular cylinder. The normalized RCS results are presented in Figs. 3 and 4 as a function of frequency for different coating materials and different orientations of the aperture. In all the figures, $\theta$ is taken as $30^{\circ}$, and the materials used for coating are shellac, natural XL ( $\epsilon_{r}=$ $3.45+0.25 i, \mu_{r}=1$ ) [21] (dashed curves), and poly-2.5dichlorostyrene $\left(\epsilon_{r}=7.3, \mu_{r}=0.91+0.32 i\right)$ [3] (solid curves). The thickness of the absorbing layer is $10 \%$ of the radius of the screen. For comparison, dotted curves represent the RCS calculated for the same CBA without any coating, and the dash-dotted line in Fig. 3(b) gives the $\mathrm{RCS}$ of a perfectly conducting circular cylinder. It is noted that for the $\varphi_{0}=180^{\circ}$ case, the average level of RCS of uncoated CBA is much higher than that of a closed uncoated circular cylinder of the same radius [see Fig. 3(b)]. In addition, strong resonances are observed in the RCS. The resonances are due to the excitation of the damped natural modes of the screen as a cavity-backed aperture. The damped modes originate from the eigenmodes of the circular cavity, $E_{m n}$, being shifted in frequency and split into even/odd pairs for which we keep the similar identifiers, $E_{m n}^{ \pm}$. In brief, this may be justified as follows. Equation (31) can be extended to complex values of $k$ and taken with a zero right-hand part, which constitutes the eigenvalue equation for natural frequencies. This equation, however, is still of the Fredholm second kind, so the natural frequencies form a discrete set of complex numbers at any value of $\theta$ [20]. However, they are continuous functions of $\theta: 0 \leq \theta<\pi$, point $\pi$ being excluded because of the logarithm term in (25). The even/odd splitting is due to breaking of eigenvalues, $E_{m n}$, of a closed cylinder, for any $m \neq 0$, by cutting the slot.

The shifted frequency locations have been calculated previously [23] for uncoated CBA. Iterative-perturbation analysis of the characteristic equation $\operatorname{det}(I-K)=0$, under assumption that $\tau=\sin (\theta / 2) \rightarrow 0$, had been carried out due to the strongly diagonal shape of the matrix. The natural frequencies are complex-valued with real parts smaller than the corresponding zeros of the Bessel 


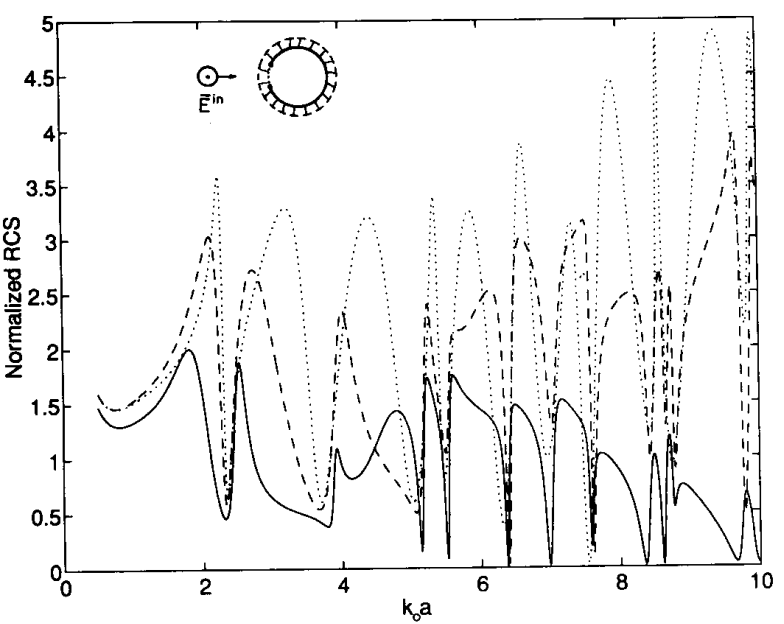

(a)

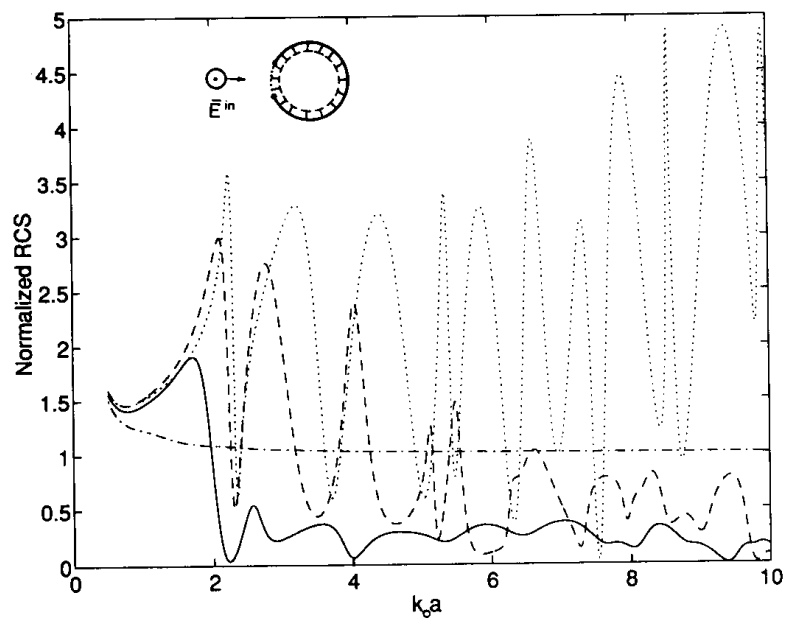

(b)

Fig. 3. The normalized RCS of (a) an uncoated and outer-coated CBA (coating radius $b=1.1 a$ ) and (b) an unslitted cylinder, uncoated and inner-coated CBA (coating radius $b=0.9 a$ ) for two different absorbing materials with CBA having $60^{\circ}$ aperture size and $\varphi_{0}=180^{\circ}$. Solid line, $\epsilon_{r}=7.3, \mu_{r}=0.91+0.32 i$; dashed line, $\epsilon_{r}=3.45+0.25 i, \mu_{r}=1$; dotted line, uncoated cylinder, i.e., $\epsilon_{r}=1, \mu_{r}=1$; dot-dashed line, unslitted cylinder.

functions. They are found as an asymptotic series

$$
\begin{array}{r}
k_{m n}^{+} a=\nu_{m n}-\frac{\delta_{m}}{2} \nu_{m n} \tau^{2}\left[1+\left(\frac{1}{2}-m^{2}+i \zeta_{m n}\right) \tau^{2}\right] \\
+O\left(\tau^{6}\right)
\end{array}
$$

for the even modes $(m=0,1,2, \ldots)$, and

$$
k_{m n}^{-} a=\nu_{m n}-\frac{1}{2} m^{2} \nu_{m n} \tau^{4}\left(1+i \gamma_{m n} \tau^{4}\right)+O\left(\tau^{10}\right)
$$

for the odd modes $(m=1,2, \ldots)$ of the empty circular

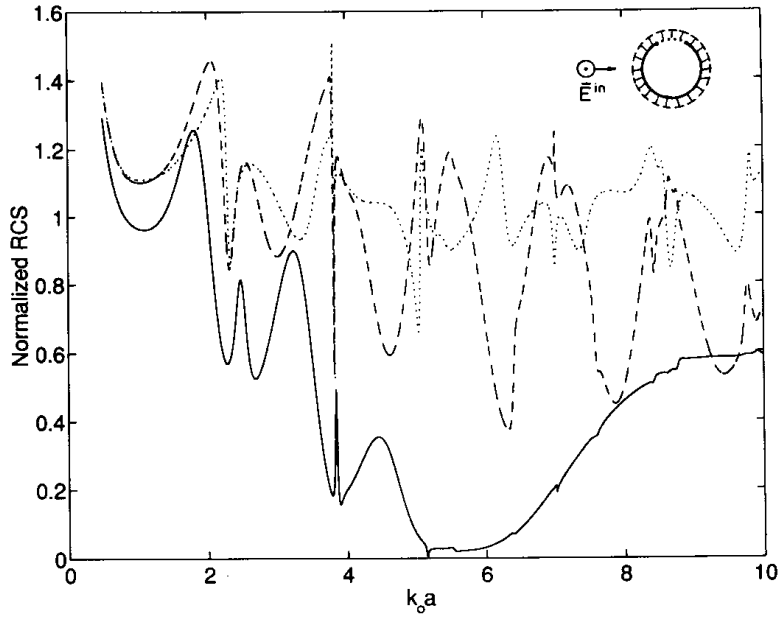

(a)

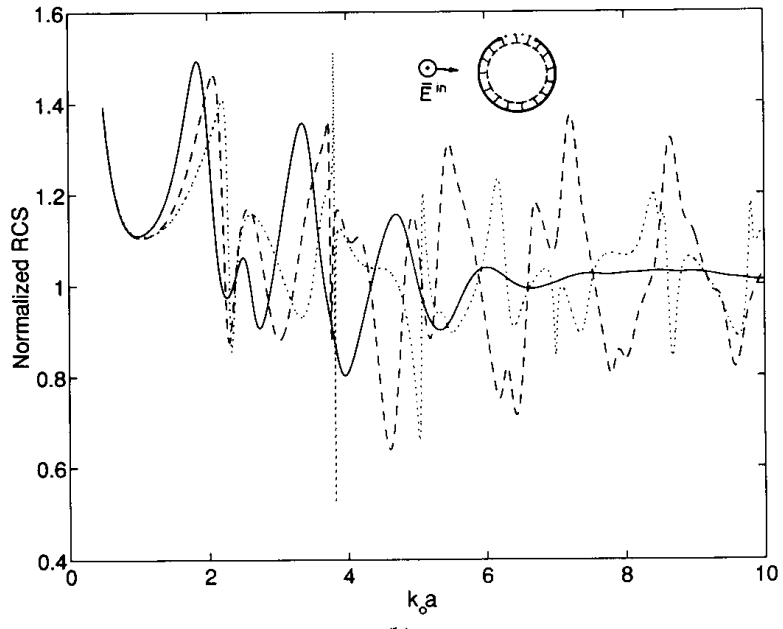

(b)

Fig. 4. The normalized RCS of (a) an uncoated and outer-coated CBA (coating radius $b=1.1 a$ ) and (b) an uncoated and inner-coated CBA (coating radius $b=0.9 a$ ) for two different absorbing materials with CBA having $60^{\circ}$ aperture size and $\varphi_{0}=90^{\circ}$. Solid line, $\epsilon_{r}=7.3, \mu_{r}=$ $0.91+0.32 i$; dashed line, $\epsilon_{r}=3.45+0.25 i, \mu_{r}=1$; dotted line, uncoated cylinder, i.e., $\epsilon_{r}=1, \mu_{r}=1$.

slitted cavity. In (37) and (38),

$$
\begin{gathered}
\zeta_{m n}=\pi^{-1} \sum_{s=0, \neq m}^{\infty} \delta_{s}\left|H_{s}\left(\nu_{m n}\right)\right|^{-2}, \\
\gamma_{m n}=\pi^{-1} \sum_{s=1, \neq m}^{\infty} s^{2}\left|H_{s}\left(\nu_{m n}\right)\right|^{-2},
\end{gathered}
$$

and $\delta_{0}=1, \delta_{s}=2$ for $s \neq 0$, and $\nu_{m n}$ is the $n$th zero of $J_{m}(x)$.

The shifted frequency locations show good agreement with the minima of RCS in the numerical results for uncoated CBA at $\varphi_{0}=180^{\circ}$. Note that for the symmetrical position of the slitted cylinder, i.e., when $\varphi_{0}=0^{\circ}$ or $180^{\circ}$, there may exist only even modes, i.e., $E_{m n}^{+} \mathrm{s}$, however for unsymmetrical cases both resonances, even and odd modes, i.e., $E_{m n}^{+}$and $E_{m n}^{-}$, do appear. The excited modes corresponding to the first four resonance frequencies for 
the $\varphi_{0}=180^{\circ}$ case are known as $E_{01}^{+}, E_{11}^{+}, E_{21}^{+}$, and $E_{02}^{+}$ [13].

The effect of the presence of the absorbing material on the outer and inner wall of the CBA are demonstrated in Fig. 3(a) and (b), respectively, for the case of aperture in the illuminated region. As observed in these figures, the lowest-order peak cannot be reduced by using absorbing dielectric material. However, when the frequency increases, the resonance peaks are reduced due to the fact that low-frequency $E$-field has a zero value on the screen and has a maximum on the axis of the cylinder, because the zeroth harmonic is dominating. However, when the frequency is increased, the number of azimuthal harmonics of comparable amplitude also increases and the location of the maximum of $E$-field moves away from the axis. Therefore, resonances of higher-order modes can be suppressed by coating the screen with the absorbing material from inside. To reduce the lowest-order resonance peak, one needs to use magnetic absorbing material as seen in Fig. 3(a) and (b). Since the magnetic field has an azimuthal component which is not zero on the screen, it can be suppressed by using lossy magnetic material, which results in a lower backscattered power. Coating from outside has no effect on the internal resonances; it only helps to decrease the amplitude of the incident field entering into the cavity. Therefore, the sharp minima cannot be suppressed, but the average level of RCS is reduced as seen in Fig. 3(a). So the resonances are just shifted in frequency but they are still sharp, which may cause the target to be easily identified.

As an example of nonsymmetrical excitation, we examine the case of $90^{\circ}$ orientation, i.e., when the aperture is looking up. Coating from the outside is very effective for reducing the average level of the RCS, but there are still sharp resonances [see Fig. 4(a)]. The frequency value at which RCS has a broad minimum in those figures corresponds to the frequency at which the reflection coefficient is minimum for quarter-wavelength magneto-dielectric coating on a perfectly conducting plane. If the coating is from the outside, some of the energy is absorbed by the coating material, so the amplitudes of the resonance peaks are reduced. On the other hand, as seen in Fig. 4(b), coating from the inside is again effective for suppressing the resonances, except the lowest one. The resonance phenomena are greatly reduced if the frequency is increased and magnetic coating is used.

The results obtained for the case when the aperture is in the shadow region are very similar to the closed cylinder case [see fig. 3(b), dash-dotted curve]. This happens because the $E$-polarized excitation induces only longitudinal current on a cylindrical scatterer, hardly reaching the shadow part of surface, and hence, not exciting the interior of CBA.

The dependence of normalized RCS on the angle of aperture orientation $\varphi_{0}$ is presented in Fig. 5 for a sample frequency, $k_{0} a=8.0$ (maximum RCS of uncoated CBA). Only lossy magnetic coating results are given for the same parameters of absorber as before. One notes again that at

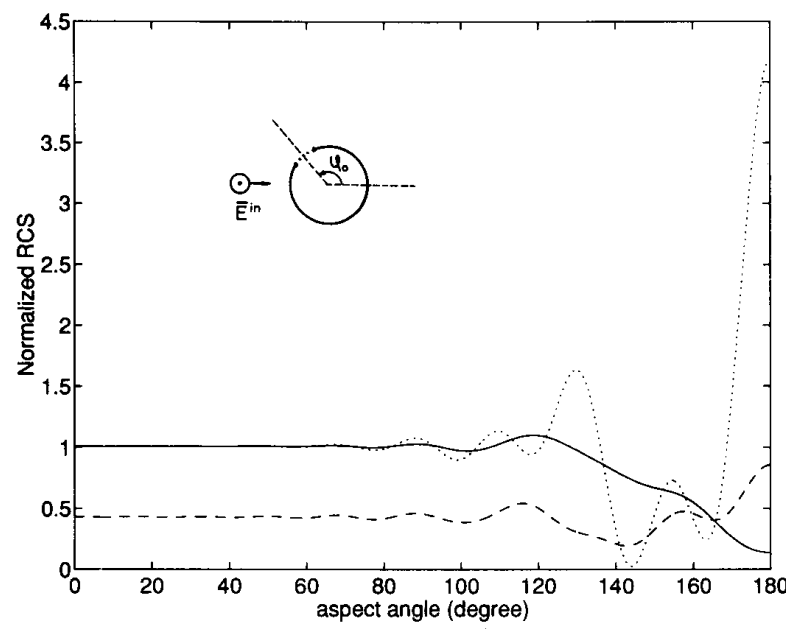

Fig. 5. The normalized RCS versus aspect angle of the screen, i.e., $\rho_{0}$, for the magnetic absorbing material with $\epsilon_{r}=7.3, \mu_{r}=0.91+0.32 i$ at $k_{0} a=8$. Solid line, inner-coated CBA; dashed line, outer-coated CBA; dotted line, uncoated cylinder, i.e., $\epsilon_{r}=1, \mu_{r}=1$.

on-aperture incidence, the inner coating (solid curves) serves much better for reducing the RCS than the outer one (dashed curves). As observed in Fig. 3(a) and (b), when the wave hits the aperture directly, the effect of resonances makes the structure strongly frequency-dependent. Therefore, RCS dependence on the angle of orientation at two different frequencies, even if they are close to each other, will be quite different.

The last two sets of graphs show the dependence of RCS on the thickness of the absorbing layer. The results are obtained only for the lossy magnetic material for coating from the inside and from the outside at some specific frequencies, namely, for $k_{0} a=1.71$ (dotted curves), 8.5 (dashed curves), and 9.39 (solid curves). As $b$ approaches $a$, RCS approaches the value for the uncoated CBA response at those frequencies [see Fig. 6(a) and (b)]. As seen from the figures, the curves have an oscillation at first and then stabilize as the thickness is increased. Mainly, there are two mechanisms involved. The first mechanism is the interference of the waves reflected from both surfaces of the material coating at the aperture, and the second mechanism is the absorption inside the coating. The former prevails when the thickness is small and the latter when it is larger. The location of the minima in Fig. 6(a) and (b) is in good agreement with the calculations for a lossy material slab of parameters $t, \epsilon_{r}$, and $\mu_{r}$, especially for higher frequencies. This validates the above explanation, since our circularly curved material coating is closer to a planar slab for higher frequencies. Hence, for practical purposes, the thickness of the dielectric slab can be chosen so that RCS has a minimum value at a specified frequency. On the other hand, if the absorbing layer were covering only the walls of CBA, but not the aperture itself, one could expect the absence of interference minima in Fig. 6(a) and (b). 


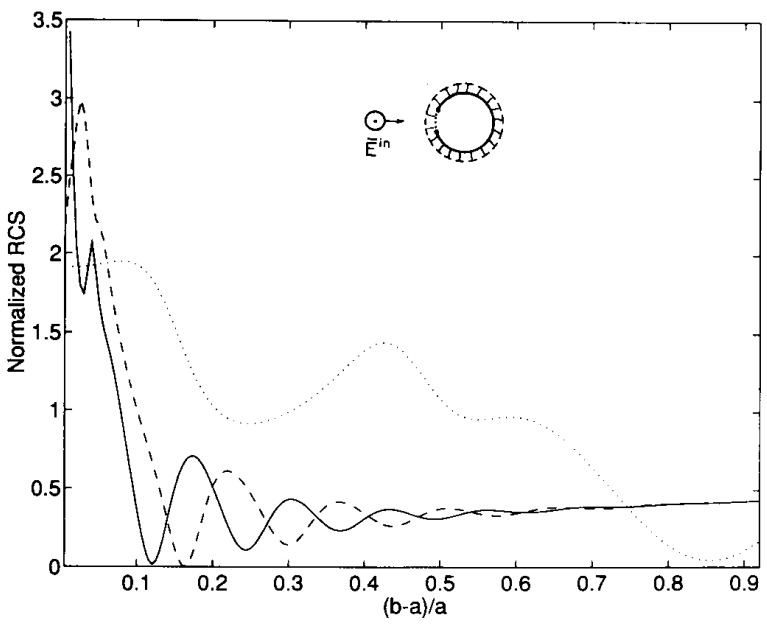

(a)

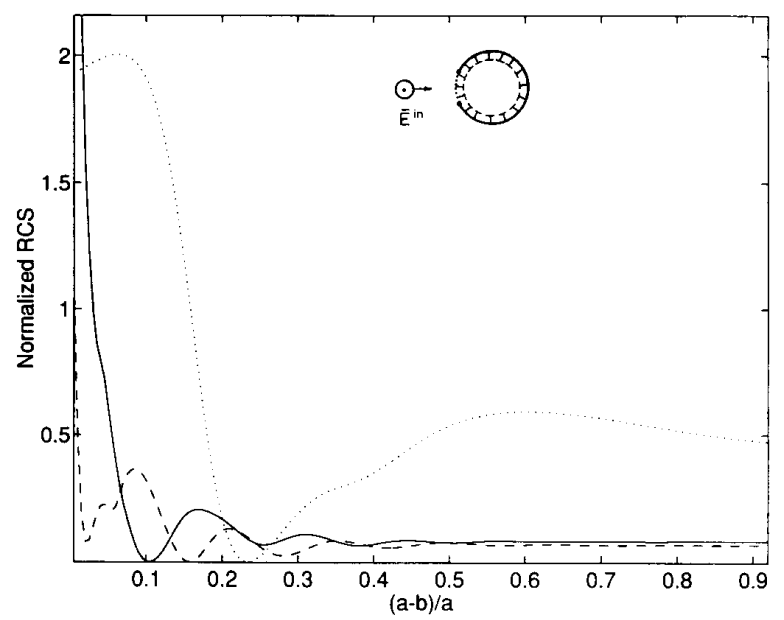

(b)

Fig. 6. The normalized RCS of (a) an outer-coated and (b) an innercoated CBA versus relative thickness of the layer. CBA has $60^{\circ}$ aperture size, $\varphi_{0}=180^{\circ}$, and $\epsilon_{r}=7.3, \mu_{r}=0.91+0.32 i$. Solid line, $k_{0} a=9.39$ dashed line, $k_{0} a=8.5$; dotted line, $k_{0} a=1.71$.

\section{CONCLUSIONS}

We have considered the problem of electromagnetic scattering of a time-harmonic plane $E$-wave from a thin, perfectly conducting, slitted infinite cylinder which is coated with absorbing material either from inside or from outside. Our aim is to study the resonances which appear in radar cross-section signatures of loaded cavities.

We first reduced the problem into dual-series equations and then solved by using the RHP technique. According to the numerical results, one can say that it is much more preferable to choose a lossy magnetic material for coating. Further, it is much better to make the coating from the inside to suppress the resonances when the interior resonance is the dominant feature in the backscattering characteristics. Moreover, in the case of direct on-aperture incidence, the inner covering is obviously better for any frequency. Otherwise, coating from the outside can also be preferable to reduce the average level of the RCS off the resonant frequency. Finally, it is possible to adjust the thickness of the absorbing layer at a specific frequency so that the RCS has a minimum value.

In this paper, the $E$-polarized incident field case is considered. The other polarization analysis results are to be reported separately.

\section{REFERENCES}

[1] A. Altıntaş, P. H. Pathak, and M. C. Liang, "A selective modal scheme for the analysis of EM coupling into or from large openended waveguides," IEEE Trans. Antennas Propagat., vol. 36, no. 1, pp. 84-96, Jan. 1988.

[2] A. Altıntaş, "Electromagnetic scattering from a class of open-ended waveguide discontinuities," Ph.D. dissertation, Dept. Elect. Eng., The Ohio State Univ., Columbus, Mar. 1986.

[3] C. S. Lee and S. W. Lee, "RCS of a coated circular waveguide terminated by a perfect conductor," IEEE Trans. Antennas Propagat., vol. AP-35, no. 4, pp. 391-398, Apr. 1987.

[4] N. H. Myung, "A high frequency analysis of electromagnetic plane wave scattering by perfectly-conducting semi-infinite parallel-plate and rectangular waveguides with absorber coated inner walls," Ph.D. dissertation, Dept. Elect. Eng., The Ohio State Univ., Columbus, 1986

[5] H. Ling, R. C. Chou, and S. W. Lee, "Shooting and bouncing rays: Calculating the RCS of an arbitrarily shaped cavity," IEEE Trans. Antennas Propagat., vol. 37, no. 2, pp. 194-205, Feb. 1989.

[6] H. Shirai and L. B. Felsen, "Rays, modes and beams for plane wave coupling into a wide open-ended parallel-plane waveguide," Wave Motion, vol. 9, pp. 301-317, 1987.

[7] K. Kobayashi, and A. Sawai, "Plane wave diffraction by an openended parallel-plate waveguide cavity," J. Electromag. Waves Appl., vol. 6, pp. $475-512,1992$.

[8] L. B. Felsen and G. Vecchi, "Wave scattering from slit coupled cylindrical cavities with interior loading: Part II-resonant mode expansion," IEEE Trans. Antennas Propagat., vol. 39, no. 8, pp. 1085-1097, August 1991.

[9] A. El-Hajj, K. Y. Kabalan, and R. F. Harrington, "Characteristic modes of a slot in a conducting cylinder and their use for penetration and scattering, TE case," IEEE Trans. Antennas Propagat., vol. 40, no. 2, pp. 156-161, Feb. 1992.

[10] Z. S. Agranovich, V. A. Marchenko, and V. P. Shestopalov, "Diffraction of a plane electromagnetic wave from plane metallic lattices," Sov. Phys. Tech. Phys., vol. 7, pp. 277-286, 1962.

[11] A. I. Nosich, "Green's function-dual series approach in wave scattering by combined resonant scatterers," in Analytical and Numerical Methods in EM Wave Theory, M. Hashimoto, $\mathrm{M}$. Idemen, and O. A. Tretyakov, eds. Tokyo: Science House, 1992.

[12] R. W. Ziolkowski, "N-series problems and the coupling of electromagnetic waves to apertures: A Riemann-Hilbert approach," Soc. Ind. Appl. Math., vol. 16, no. 2, 1985.

[13] R. W. Ziolkowski and J. B. Grant, "Scattering from cavity-backed apertures: The generalized dual series solution of the concentrically loaded E-pol slit cylinder problem," IEEE Trans. Antennas Propagat., vol. AP-35, no. 5, pp. 504-528, May 1987.

[14] W. A. Johnson and R. W. Ziolkowski, "The scattering of an $H$-polarized plane wave from an axially slotted infinite cylinder: A dual series approach," Radio Sci., vol. 19, no. 1, pp. 275-291, Jan.-Feb. 1984.

[15] J. R. Mautz, and R. F. Harrington, "EM penetration into a conducting circular cylinder through a narrow slot, TM case," J. Electromag. Waves Appl., vol. 2, no. 3/4, pp. 269-293, 1988.

[16] O. G. Gamulya and A. I. Nosich, "Scattering cross sections and ponderomotive forces at the diffraction of $E$-polarized plane wave from an open circular screen," Preprint No. 326. Kharkov: IRE-Kharkov Press, 1986 (in Russian).

[17] T. M. Wang and H. Ling, "Resonant behavior of conducting slotted rectangular shell," Microwave Opt. Tech. Lett., vol. 1, pp. 320-323, Nov. 1988

[18] P. M. Goggans, and T. H. Shumpert, "Backscatter RCS for TE and TM excitations of dielectric-filled cavity backed apertures in 2-D bodies," IEEE Trans. Antennas Propagat., vol. 39, no. 8, pp. 1224-1227, Aug. 1991. 
[19] M. Abramowitz and I. A. Stegun, Handbook of Mathematical Functions. New York: Dover, 1965.

[20] S. Steigberg, "Meromorphic families of compact operators," Arch. Rational Mech. Anal., vol. 31, no. 5, pp. 372-379, 1968.

[21] R. F. Harrington, Time-Harmonic Electromagnetic Fields. New York: McGraw-Hill, 1961.

[22] P. M. Morse and H. Feshbach, Methods of Theoretical Physics, vol 1. New York: McGraw-Hill, 1953, Sec. 8.2, pp. 907-925.

[23] A. I. Nosich, "Electromagnetic characterization of unclosed circular cylindrical screens," Ph.D. Dissertation, Kharkov University, 1979 (in Russian).

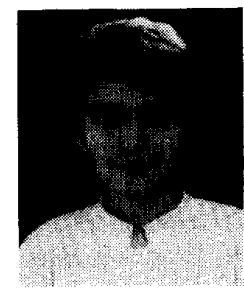

sembly in 1993.
Dilek Çolak was born in Aksehir, Turkey, in 1970. She received her B.S. and M.S. degrees in electrical engineering from Bilkent University, Ankara, Turkey, in 1991 and 1993, respectively, where she has been a research assistant since 1991. Her research interests include analytical and numerical solution of electromagnetic scattering problems and radar cross section analysis.

She was one of the recipients of the Young Scientist Award of the International Union of Radio Science (URSI) at the 24th General As-

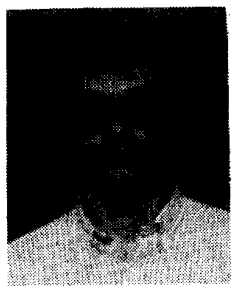

Alexander I. Nosich was born in 1953 in Kharkov, Ukraine. Had graduated from the Radio Physics Department of Kharkov University in 1975 . He has received his Ph.D. and D.Sc. degrees in radio physics from Kharkov University, in 1979 and 1990 , respectively. Since 1978 , he has been with the Electronics Department of the Institute of Radiophysics and Electronics of the Ukrainian Academy of Sciences, Kharkov, as a junior and senior research scientist. From February to $\mathrm{Au}-$ gust 1992, he was with the Department of Elec trical and Electronics Engineering of the Bilkent University, Ankara, Turkey, as a visiting professor through the Fellowship of Turkish Scien- tific and Technical Research Council (TÜBİTAK). From October 1992 to March 1993, he was with the Department of Electrical Engineering and Computer Science of Kumamoto University, Japan, as a guest scientist through the Fellowship of the Matsumae International Foundation. Currently, he is a visiting associate professor of the same university. Dr. Nosich's interests are in free space and open waveguide scattering, complex modes behavior, radar cross-section modifying, and antenna simulation.

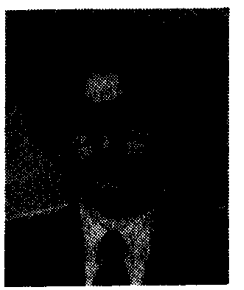

Ayhan Altıntas was born on March 29, 1958 in Turkey. He received his B.S. and M.S. degrees from the Middle East Technical University (METU), Ankara, Turkey, in 1979 and 1981, respectively, and the Ph.D. degree from The Ohio State University, Columbus, in 1986, all in electrical engineering.

From 1981 to 1987 , he was with the ElectroScience Laboratory, The Ohio State University, first as a graduate research associate and then as a post-doctoral researcher. Afterward, he has spent one year at the Optical Sciences Center of The Australian National University, Canberra, Australia. Currently, he is an associate professor at the Department of Electrical and Electronics Engineering, Bilkent University, Ankara, Turkey, where he was an assistant professor from February 1988 to May 1990 . His research interests are in electromagnetic radiation and scattering, microwaves, fiber optics, and integrated optics.

Dr. Altıntas is a member of Sigma Xi and Phi Kappa Phi, He is the Chairman of IEEE Turkey section for the term 1991-1993. He is the recipient of The Ohio State University, ElectroScience Laboratory Outstanding Dissertation Award of 1986, IEEE 1991 Outstanding Student Branch Counselor Award, and the 1991 Research Award of the Professor Mustafa N. Parlar Foundation of METU. 